\title{
6 ADHS und Epilepsie
}

\author{
Christine Ettrich
}

Die Kombination von ADHS und Epilepsie wurde bislang in der Fachliteratur zu wenig beachtet. Besonders der unaufmerksame Typus (ADS) wird häufig übersehen. Jugendliche Epileptiker mit ADHS sind deutlich schlechter integriert und versagen häufiger in der Schule und in der Berufsausbildung. Eine differenzierte Diagnostik und die Berücksichtigung der Komorbidität bei der Therapie ist mit positiven Konsequenzen für die Prognose dieser Patienten verbunden.

\subsection{Problemlage}

Obgleich in der Literatur der Zusammenhang zwischen Kognition und Epilepsie seit Langem bekannt ist (Finck et al. 1995, Gross-Tsur et al. 1997, Siemes et al. 2001), wird der Kombination der beiden Störungsbilder ADHS und Epilepsie bislang zu wenig Beachtung geschenkt. Dabei ist davon auszugehen, dass in einer Epilepsie-Spezialsprechstunde etwa 25\% der Patienten komorbid an einer $\mathrm{AD}(\mathrm{H})$ S leiden (Ettrich 2006, Stollhoff et al. 2004, Stollhoff und Schulte-Markwort 2004). Andererseits wurde in einer kürzlich erschienenen Studie an 68 Kindern gezeigt, dass Kinder, die wegen einer ADHS in Behandlung kamen, in 7,3\% der Fälle auch eine Epilepsie hatten (Takashi et al. 2003). Es handelt sich also durchaus nicht um eine seltene Kombination zweier Störungen, da die ADHS im Kindes- und Jugendalter ja recht häufig auftritt ( 2 bis $6 \%$ bei Kindern und Jugendlichen im Alter zwischen sechs und 18 Jahren) und die Epilepsie ja im Kindes- und frühen Jugendalter einen ihrer beiden Häufigkeitsgipfel aufweist. 


\subsection{Wie ist die Kombination beider Störungen zu erklären?}

Seit Langem ist bekannt, dass die von Epileptikern häufig gezeigten Verhaltensauffälligkeiten nicht eine Reaktion des Organismus auf die stattgefundenen bzw. stattfindenden epileptischen Anfälle darstellen, sondern dass sie gemeinsam mit den epileptischen Anfällen als Ursache eine hirnorganische Vulnerabilität haben, beides also Symptome ein und derselben Grunderkrankung sind. Aus Studien (Takashi 1996, Barkley 1998) geht hervor, dass beiden Störungen auch eine gemeinsame genetische Komponente zugrunde liegen kann. Bei beiden Störungen finden wir eine erhöhte Gamma-Aktivität (3o bis $80 \mathrm{~Hz}$ ), was für eine erhöhte Erregbarkeit des Kortex spricht. Die Gamma-Aktivität korreliert mit der Ausprägung kognitiver Merkmale.

Dies kann die schulischen und daraus folgenden sozialen Probleme bei komorbid erkrankten Kindern und Jugendlichen erklären. Formen der idiopathischen fokalen Epilepsie, besonders solche, die mit einem bioelektrischen Status einhergehen (ESES), manifestieren sich häufig unter dem klinischen Bild einer ADHS, meist kombiniert mit deutlichen kognitiven Störungen (Herrmann 2005, Holtmann et al. 2003, Jung et al. 2000).

\subsection{Welche diagnostischen Schlussfolgerungen sind hieraus zu ziehen?}

Als Schlussfolgerung für die Diagnostik ergibt sich hieraus, dass bei Kindern mit Epilepsie besonders beim Auftreten schulischer und sozialer Probleme immer auch an ADHS zu denken ist und entsprechend eine Diagnostik zu erfolgen hat. Besondere Sorgfalt ist bei progredienten und multiplen Entwicklungsstörungen geboten. Immer dann, wenn mehrere Störungsbilder kombiniert auftreten, ist es wichtig, eine saubere und umfassende Diagnostik durchzuführen, um die Anteile der einzelnen Störungen am Gesamtbild des Patienten möglichst genau zu erkennen. Dies dient schließlich der Optimierung der anzuwendenden therapeutischen Optionen.

\subsection{Antiepileptika und ADHS-Medikamente - synergistisch oder antagonistisch wirksam?}

In der medikamentösen Therapie von Epilepsien haben sich eine Vielzahl von Antiepileptika mit unterschiedlichem Wirkspektrum für die einzelnen Anfallsarten bewährt. So ist es heute möglich, durch eine „taylor-made-therapie“ mit Antikonvulsiva ca. 60-80\% der Patienten anfallsfrei oder zumindest wesentlich anfallsärmer zu bekommen.

Als Goldstandard in der medikamentösen Behandlung der ADHS gilt nach wie vor das Methylphenidat in seinen unretardierten und retardierten Formen. Auch hier gelingt es bereits ganz gut, die medikamentöse Therapie den individuellen Erfordernissen des jeweiligen Patienten anzupassen. 
Was aber, wenn beide Störungsbilder kombiniert bei demselben Patienten auftreten? Methylphenidat gehört zu den Stimulanzienpräparaten. Es ist bekannt, dass es z. B. die Verstoffwechselung von Phenobarbital, Phenytoin und Primidon hemmt. Stimulanzien können prinzipiell, auch das ist bekannt, die Anfallsschwelle senken und könnten damit ein epileptisches Anfallsleiden verschlimmern. Ist demzufolge in der Therapie von Epilepsiepatienten mit ADHS auf Stimulanzien zu verzichten oder unter welchen Bedingungen kann eine optimale Behandlung beider kombiniert auftretender Störungen erreicht werden?

Das ist die in der Gegenwart in vielen Diskussionen in Fachkreisen immer wieder auftretende Frage. Die Studienlage dazu ist insgesamt noch sehr dünn, viele Empfehlungen fußen auf dem klinischen Eindruck. Insbesondere die Anzahl der prospektiven Studien bedarf einer deutlichen Erweiterung, um verlässliche Aussagen zu erlauben. Eine präzise Risikoabschätzung fällt demzufolge gegenwärtig aufgrund der noch deutlich zu geringen Datenlage noch schwer.

Allerdings scheint Methylphenidat nur über ein geringes pro-konvulsives Potenzial zu verfügen. So konnte in einer Studie von Gucuyener et al. an 57 Kindern nachgewiesen werden (Gucuyener et al. 2003), dass lediglich 5 davon nach zusätzlicher Einnahme von Methylphenidat zu den bereits verabreichten Antiepileptika eine Anfallsaktivierung aufwiesen. In den wenigen offenen prospektiven Studien, die alle eine recht kleine Patientenzahl (zwischen 9 und 23) aufwiesen (Feldmann et al. 1989, Finck et al. 1995, Gross-Tsur et al. 1997), zeigte sich, dass zum einen keine Zunahme von Anfällen nach zusätzlicher Gabe von Methylphenidat erfolgte, in den meisten Fällen auch keine vermehrte paroxysmale EEG-Aktivität zu finden war. In einer weiteren Studie (Ernst et al. 1999) fand sich sogar ein Verschwinden paroxysmaler EEG-Aktivität bei klinisch anfallsfreien Kindern nach Zugabe von Methylphenidat. Bei medikamentös gut eingestellten kindlichen und jugendlichen Epilepsiepatienten scheint also die zusätzliche Einnahme von Methylphenidat in den Fällen, in denen eine ADHS diagnostisch gesichert wurde, positive Effekte zu haben.

Einer besonderen Betreuung bedürfen diejenigen Epilepsiepatienten, die unter Antikonvulsivagabe noch nicht anfallsfrei geworden sind und dennoch eine ADHS von einem Störungswert aufweisen, der eine medikamentöse Mitbehandlung notwendig macht. Nach der derzeitigen Datenlage sollte auch hier nicht auf eine Methylphenidatgabe verzichtet werden, allerdings ist das Monitoring entsprechend eng und individuell zu halten, d.h. diese Kinder und Jugendlichen bedürfen einer besonders sorgfältigen Begleitdiagnostik und Überwachung.

Eine weitere wichtige Frage ist die nach der Zugabe von Methylphenidat zu einer bereits kombinierten antiepileptischen Therapie mit unterschiedlichen Antikonvulsiva. Natürlich scheint der Schluss nahe zu liegen, dass der erste Schritt in Richtung Monotherapie der Epilepsie gehen sollte. Andererseits wissen wir, dass dies in praxi nicht immer zu erreichen ist. Hier sind aufgrund der dünnen Studienlage ebenfalls noch keinerlei Aussagen möglich. 


\subsection{Welche spezielle Kombination beider Störungen lässt gegenwärtig den sichersten Erfolg einer medikamentösen Kombinationstherapie erwarten?}

Interessant und vor dem Hintergrund früherer Arbeiten erneut bemerkenswert ist die Tatsache, dass bei Patienten mit kindlicher Absence-Epilepsie und juveniler myoklonischer Epilepsie teilweise eine deutliche Verbesserung unter der Zugabe von Methylphenidat zu verzeichnen war (Takashi et al. 2003). Hierfür könnte auch die „Nähe“ des klinischen Erscheinungsbildes eine Rolle spielen (Krause und Krause 200o). Interessant wäre es auch, die Wirkung von Carbamazepin, das ja inzwischen sowohl in der Antiepileptologie als auch in der Psychiatrie eingesetzt wird, bei Kindern mit kombinierter Störung Epilepsie/ ADHS systematisch zu untersuchen, da hier möglicherweise mit dem Carbamazepin sehr effektiv gearbeitet werden kann (Behar et al. 1998). Andererseits ist bei dem Einsatz von Carbamazepin bei alleiniger ADHS aufgrund des Nebenwirkungsprofils dieses Präparates weiterhin Zurückhaltung geboten.

\subsection{Welche Parameter bedürfen einer Verlaufskontrolle?}

Vor Beginn einer Behandlung mit Methylphenidat sollte in jedem Fall ein EEC abgeleitet werden, das im Verlauf der Behandlung durch Kontrollableitungen ergänzt wird (Schmidt et al. 2002). Auch bei auffälligem EEG ohne bisherige klinische Anfälle bedarf die Methylphenidattherapie einer Überwachung mittels EEG-Kontrolle. Bei schlecht kontrollierter Epilepsie ist zunächst die Bestrebung darauf zu richten, die antikonvulsive Einstellung zu optimieren, bevor die ADHS mit Methylphenidat behandelt wird (Krause und Krause 2000, Stollhoff 2006). Allerdings wissen wir aus der Praxis, dass sich mitunter auch die Therapieeffekte verschiedener Präparate ergänzen können, so dass wiederum auch nicht zu lange mit der Behandlung der ADHS gewartet werden sollte. Stollhoff (Stollhoff 2006) berichtet, dass nach Aussagen von Eltern und Lehrern $82 \%$ der sowohl antikonvulsiv als auch mit Methylphenidat behandelten Kinder eine deutliche Verbesserung der Aufmerksamkeit und der sozialen Integration erfuhren.

\subsection{Wie geht es jenseits des Kindes- und Jugendalters weiter?}

Ein nicht zu vernachlässigender Gesichtspunkt in diesem Problemfeld ist, dass die ADHS sich ja in ein bis zwei Drittel der Fälle bis ins Erwachsenenalter hält, dass also, wie in den letzten Jahren verstärkt berichtet wurde, auch Erwachsene unter dieser Störung leiden (Wender 1997, Wender 1995), was eine Mitbehandlung durch Methylphenidat auch im Adoleszenten- und Erwachsenenalter erforderlich macht, will man die kognitive und soziale Entwicklung der Patienten positiv beeinflussen und zu ihrer besseren sozialen Integration beitragen (Krause et al. 1998, Krause et al. 1999). Dem steht entgegen, dass Met- 
hylphenidat gegenwärtig für die Behandlung Erwachsener noch nicht zugelassen ist (Adam et al. 1999).

Hier liegt ein weiteres Arbeitsfeld für Forschung und Praxis gleichermaßen, wenn man diesem Patientenkreis künftig besser gerecht werden will.

\section{Zusammenfassung}

Die von vielen Ärzten gezeigte generelle Zurückhaltung bei der Verordnung von Methylphenidat an anfallskranke Patienten mit komorbider ADHS ist nicht berechtigt. Wir brauchen andererseits mehr kontrollierte (prospektive) Studien. Es wird künftig notwendig sein, den Inhalt der Beipackzettel von Methylphenidat-Präparaten entsprechend moderner Erkenntnisse zu aktualisieren.

\section{Literatur}

Adam C, Döpfner M, Lehmkuhl G. Pharmakotherapie hyperkinetischer Störungen im Erwachsenenalter. Fortschr. Neurol. Psychiatr. 1999; 67: 359-366.

Aicardi J. Epilepsy as a non-paroxysmal disorder. Acta Neuropediatr. 1996; 2: 249-257.

Barkley RA. Attention-deficit hyperactivity disorder. New York, London: Guilford Press 1998.

Behar D, Schaller J, Spreat S. Extreme reduction of methylphenidate levels by carbamazepine. J. Am. Acad. Child Adolesc. Psychiatry 1998; 37: 1128-1129.

Ernst E, Pettenburger K, Gößler R et al. Zur Wirkung von Methylphenidat bei Patienten mit kindlichen Absencen und einer Aufmerksamkeitsstörung. Epilepsieblätter - Praktische Epileptologie, Suppl. 1999; 12: 58.

Ettrich C. Komorbide Störungen bei Epilepsie. Vorlesungsskript. Universität Leipzig. 2006.

Feldmann H, Crumrine P, Handen BL et al. Methylphenidate in children with seizures and attention-deficit disorder. Am. J. Dis. Child 1989; 143: 1081-1086.

Finck S, Metz-Lutz MN, Bécache E et al. Attention-deficit hyperactivity disorder in epileptic children: a new indication for methylphenidate? Ann. Neurol. 1995; 38: 520.

Gross-Tsur V, Manor 0, van der Meere J et al. Epilepsy and attention deficit hyperactivity disorder: Is methylphenidate safe and effective? J. Pediatr. 1997; 130: 670-674.

Gucuyener K, Erdemoglu AK, Senol S, Serdaroglu A, Soysal S, Kockar Al. Use of methylphenidate for attentiondeficit hyperactivity disorder in patients with epilepsy or electroencephalographic abnormalities. I Child Neurol. 2003; 18 (2): 109-112.

Herrmann CS. Die psychopathologische Bedeutung hochfrequenter EEG-Oszillationen. Zeitschrift für Neuropsychologie.2005 16 (3): 151-162.

Holtmann M et al. Increased Frequency of Rolandic Spikes in ADHD Epilepsia. 2003; 44: 1241-1244.

Jung $\mathrm{M}$ et al. Das ESES-Syndrom in der Kinder- und Jugendpsychiatrie. Z. Kinder Jugendpsychiatr Psychother. 2000; 28 (1): 17-24.

Krause KH, Krause I, Trott GE. Das hyperkinetische Syndrom (Aufmerksamkeitsdefizit-/Hyperaktivitätsstörung) im Erwachsenenalter. Nervenarzt 1998; 69: 543-556.

Krause KH, Krause |, Trott GE. Diagnostik und Therapie der Aufmerksamkeitsdefizit-/Hyperaktivitätsstörung im Erwachsenenalter. Dtsch. med. Wschr. 1999; 124: 1309-1313.

Krause, KH, Krause J. Ist die Gabe von MPH bei Komorbidität von Epilepsie und $\mathrm{AD}(\mathrm{H}) \mathrm{S}$ kontraindiziert oder nicht? Akt. Neur. 27, 2000; 72-76.

Schmidt JK, Pluck I, von Gontard A. Waived EEG diagnosis before administration and during drug therapy with methylphenidate: dangerous or justifiable? Z Kinder Jugendpsychiatr Psychother. 2002; 30 (4): 295-302.

Siemes $\mathrm{H}$ et al. Anfälle und Epilepsie bei Kindern und Jugendlichen. Thieme 2001.

Stollhoff K et al. Koinzidenz von Epilepsie und ADHS. Kinder- und Jugendarzt 2004; 35 (5):197-202.

Stollhoff K, Schulte-Markwort M. ADHS und assoziierte Störungen. Pädiatrie Hautnah. 2004; 7. 


\section{ADHS und Epilepsie}

Stollhoff K. Epilepsie und ADHS - eine seltene Kombination? Pädiatrie Hautnah. 2006; 3.

Takashi I et al. Comorbidity in ADHD. Psychiatry and clinical sciences 2003; 57: 457-463.

Tugendhaft P. Clinical and EEG evidence for antiepileptic efficacy of methylphenidate in juvenile myoclonic epilepsy. Epilepsia (Suppl 5) 1996; 37: 174.

Wender PH. Attention-deficit hyperactivity disorder in adults - a wide view of a widespread condition. Psychiatric Ann. 1997; 27: 556-562.

Wender PH. Attention-deficit hyperactivity disorder in adults. Oxford, New York: Oxford University Press 1995. 